\title{
Global cloud-permitting simulations of Typhoon Fengshen (2008)
}

\author{
Tomoe Nasuno 1* (10, Hiroyuki Yamada², Masuo Nakano ${ }^{1}$, Hisayuki Kubota ${ }^{3}$, Masahiro Sawada ${ }^{4}$ and Ryuji Yoshida ${ }^{5}$
}

\begin{abstract}
Large-scale fields and inner-core processes relevant to the formation and intensification of Typhoon Fengshen (2008) were examined by simulations using a global nonhydrostatic model with a cloud-permitting resolution. Five runs were performed by varying the cloud microphysics or initial condition settings. In three out of five runs, a middle tropospheric trough developed within a few days following a large-scale latent heat release, which enabled the successive occurrence of deep convective events within the 50-km radius of the incipient disturbance and subsequent tropical cyclone (TC) formation. In the run initialized by altering the analysis dataset, collocation between latent heat release and the large-scale gyre was less evident, and neither the trough nor a TC developed. In the run with weaker latent heating in the lower troposphere, the trough was weak and TC formation was significantly delayed. These results indicate that the superposition of large-scale disturbances in the lower and middle troposphere and their linkage through convective enhancement played an important role in the genesis of Fengshen by preconditioning the establishment of a deep upright inner core.
\end{abstract}

Keywords: Tropical cyclone, global nonhydrostatic model, Cloud-permitting simulation

\section{Background}

The genesis of a tropical cyclone (TC) in the western North Pacific (WNP) occurs under influences of a wide range of spatio-temporal variabilities. The summertime monsoon trough provides favorable background conditions for a TC precursor to develop through multi-scale interactions (Holland 1995). The Madden-Julian Oscillation (MJO; Madden and Julian 1971) or boreal summer intraseasonal variability (BSISO) (e.g., Wang and Rui 1990) has positive influences on TC genesis in the convectively active period (Liebmann et al. 1994; Maloney and Hartmann 2001; Schreck and Molinari 2011; Hsu et al. 2011; Yoshida et al. 2014). Easterly waves, mixed Rossby-gravity (MRG) waves, and tropical depression (TD)-type disturbances often serve as TC precursors (Yanai 1961; Frank and Roundy 2006; Ritchie and Holland 1999; Schreck et al. 2011; Dickinson and Molinari 2002; Zhou and Wang 2007). The TD-type disturbance

\footnotetext{
*Correspondence: nasuno@jamstec.go.jp

1 Japan Agency for Marine-Earth Science and Technology, 3173-25

Showa-machi, Kanazawa-ku, Yokohama, Kanagawa 236-0001, Japan Full list of author information is available at the end of the article
}

is dynamically close to the synoptic-scale wave train (SWT) that often appears before and after TC genesis, with a zonal wavelength of $2500-3500 \mathrm{~km}$, northeastsouthwest oriented phase tilt, and northwestward phase propagation. Middle- to upper-tropospheric disturbances sometimes play an important role in TC genesis through the enhancement of upper-level divergence or intensification of middle tropospheric vorticity (Sadler 1976; Briegel and Frank 1997; Heta 1990; Ritchie and Holland 1997; Tam and Li 2006; Zhou and Wang 2007). On the other hand, the critical role of diabatic processes in TC genesis has been broadly recognized (e.g., review by Houze 2010), which often appears as "mesoscale convective systems (MCSs)" (Bister and Emanuel 1997; Ritchie and Holland 1999) or "hot towers" (Simpson et al. 1998; Hendricks et al. 2004). Based on numerical experiments with resolving mesoscale convection (MC), Yamasaki (1983) demonstrated the importance of successive formation of MC and its interaction with stormscale fields, especially in the early formation stage of TCs.

Specific aspects of the above-mentioned factors have been well documented by previous studies (e.g., Fu 
et al. 2007; Kerns and Chen 2013; Maloney and Hartmann 2001; Nolan 2007; Ritchie and Holland 1999; Yoshida and Ishikawa 2013; Xu et al. 2013, 2014; Wu and Duan 2015). In the individual cases of TC genesis, however, multiple factors simultaneously have an influence, and may interact among themselves. To further deepen our understanding of this issue, it is useful to examine the combination of multiple factors by detailed case studies.

A global cloud-permitting simulation is one of useful methods for such an investigation. This approach has the merit of representing a broad range of scale interactions from mesoscale cloud systems to planetary scale variabilities in a seamless framework (Kodama et al. 2015; Nakano et al. 2015; Yamada et al. 2016). It requires fewer assumptions to represent moist convection than conventional general circulation models by explicit treatment and is also free from lateral boundary problems in regional models (Warner et al. 1997), although the model does not necessarily outperform convectional general circulation models or regional nonhydrostatic models in all aspects due to its own biases (Kodama et al. 2015).

In this study, we focused on Typhoon Fengshen (2008), which was formed during the onset of the WNP monsoon (Geng et al. 2011) and in an active phase of BSISO (Katsumata et al. 2013). Based on an examination of the European Center for Medium-Range Weather Forecasts (ECMWF) year of tropical convection (YOTC) operational analysis (Waliser et al. 2012; hereafter ECMWF YOTC), Xu et al. (2013) categorized the synoptic-scale precursor of Fengshen as the "SWT" type, whereas Yoshida and Ishikawa's (2013) index, based on the 850hPa flow pattern, classified Fengshen as the "monsoon confluence" type.

Figure 1 shows the atmospheric conditions during the genesis of Fengshen in the ECMWF YOTC and the National Centers for Environmental Prediction (NCEP) final analysis (hereafter NCEP FNL). Both sets of data represent a low-level westerly over the equatorial western Pacific and a large-scale cyclonic gyre associated with the BSISO event (Fig. 1a, b), and moisture was accumulated within the gyre (Fig. 1c, d). Interestingly, differences are found between the analyses in moisture and flow fields that might affect convective behavior. Under these largescale conditions, Yamada et al. (2012), who analyzed the in situ observation and cloud-permitting simulation data, revealed the critical role of the vertical superposition of mesoscale convective vortices for this case. Thus, Fengshen is a suitable target for an investigation of the multiple factors at different scales and the convective effects on the TC. In this study, we investigated these issues using a coordinated set of simulations.

\section{Methods \\ Numerical model}

Nonhydrostatic Icosahedral Atmospheric Model (NICAM; Satoh et al. 2008, 2014) was used for the simulation in this study. The horizontal mesh size was $3.5 \mathrm{~km}$ ("cloud-permitting") and 40 vertically uneven levels were taken. Moist processes were explicitly calculated with the NICAM Single-moment Water 6 scheme (NSW6; Tomita 2008), and no cumulus parameterization was used. The atmospheric turbulence and radiation processes were calculated using a modified version of the Mellor-YamadaNakanishi-Niino level 2 scheme (Mellor and Yamada 1982; Nakanishi and Niino 2006; Noda et al. 2010) and Model Simulation radiation TRaNsfer code (MSTRN) X (Sekiguchi and Nakajima 2008), respectively. The land surface process was represented by a bucket model. Sea surface temperature (SST) was predicted using a slab ocean model, and was nudged to the weekly mean observations from National Oceanic and Atmospheric Administration (NOAA) Optimum Interpolation Sea Surface Temperature (Reynolds et al. 2002) with a relaxation time of 5 days.

\section{Experimental setup}

In this study, four simulations were performed with initial data interpolated from the $0.5^{\circ}$ gridded ECMWF YOTC, with varying cloud microphysical settings (Table 1) to determine their impacts on the evolution of the largescale variability and the initial disturbance. In "E_mp1," sedimentation of cloud ice was included, as was the case in the $3.5-\mathrm{km}$ mesh simulation of Fengshen (Hashino et al. 2013, 2016; Yamada et al. 2016). In "E_mp2," the default setting was used (the same as Nasuno 2013). In "E_mp3," the upper-tropospheric clouds were tuned for cloud-radiative balance in the 14- $\mathrm{km}$ mesh multi-decadal simulations (Kodama et al. 2015). In "E_mp4," the terminal velocity of precipitating condensates was reduced, which was tuned for 14- $\mathrm{km}$ mesh ensemble simulations of MJO events (similar to Miura et al. 2015). The impact of atmospheric conditions (Fig. 1; Additional file 1: Figure S1) was examined by the "N_mp2" run, where the $1.0^{\circ}$ gridded NCEP FNL was used to create the initial data, with other settings the same as in "E_mp2." The thermal conditions were more stabilized in the NCEP FNL than in the ECMWF YOTC, with more humidity in the free atmosphere (Additional file 1: Figure S1a, b). In all runs, the land surface and the slab ocean model were initialized using the NCEP FNL. The simulation period was 

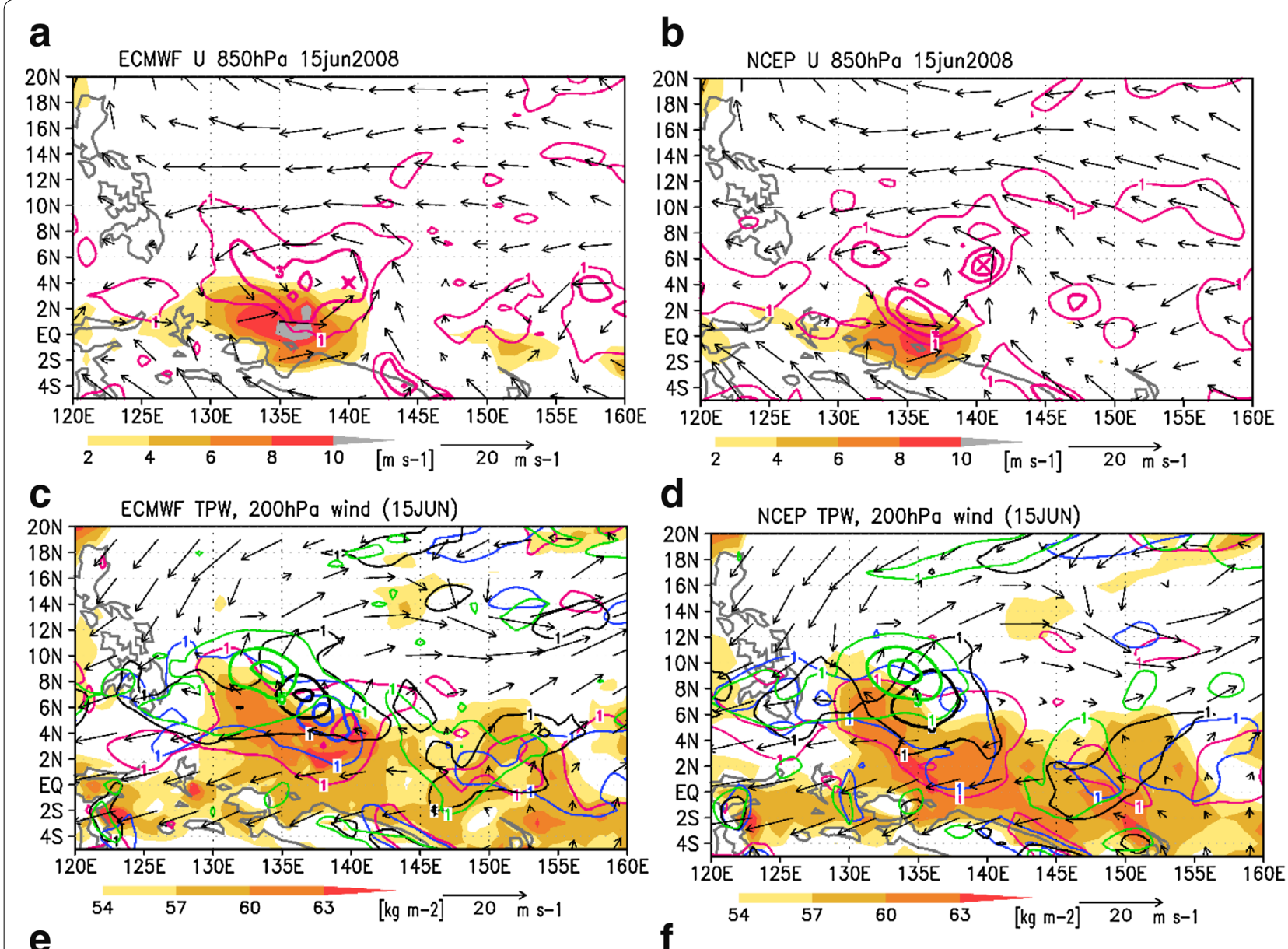

e
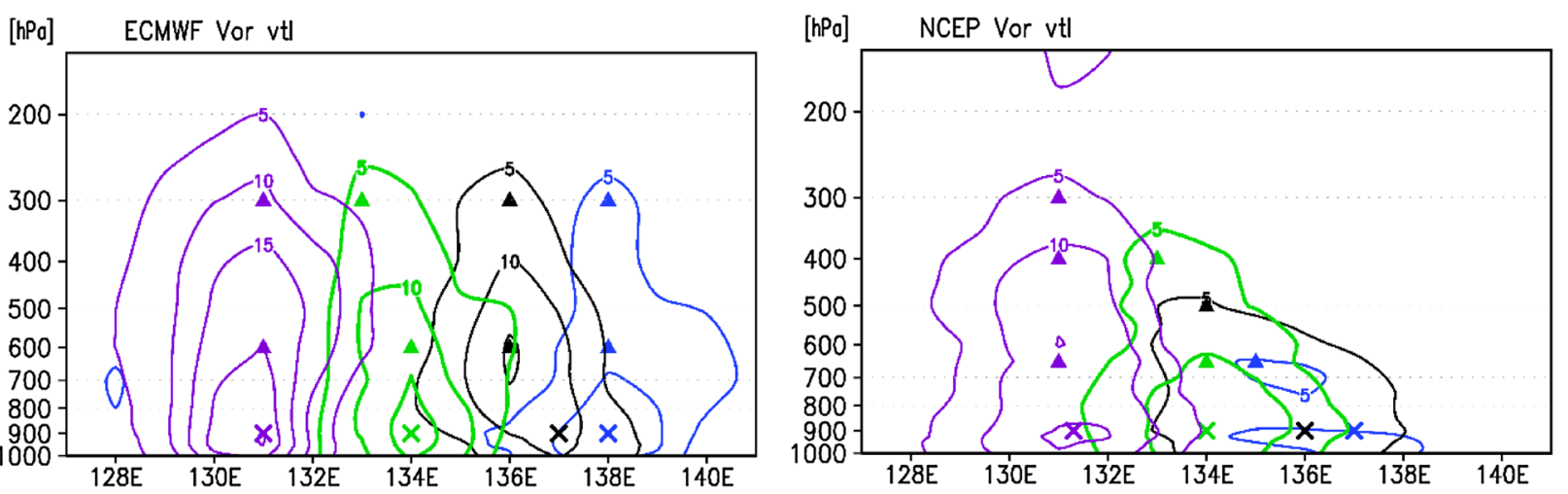

Fig. 1 a, b Zonal velocity (shading), relative vorticity (red contour; 1, 3, and $5 \times 10^{-5} \mathrm{~s}^{-1}$ ), and wind vectors at $850 \mathrm{hPa}$, c, $\mathbf{d}$ column-integrated water vapor (shading) and 200-hPa wind vectors, at 0000 UTC on 15 June 2008, e, f height-longitude section of relative vorticity at 0000 UTC on 16 (blue), 17 (black), 18 (green), and 19 (purple) June 2008, along the latitude across the center of the incipient disturbance, in the ECMWF YOTC (a, c, e) and the NCEP FNL (b, d, f). Contour lines of 850-hPa relative vorticity averaged between 6 hours before and after 0000 UTC on 15 (red), 16 (blue), 17 (black), and 18 (green) June 2008 are also depicted in $\mathbf{c}, \mathbf{d}\left(1,5\right.$, and $\left.10 \times 10^{-5} \mathrm{~s}^{-1}\right)$. Crosses in $\mathbf{a}, \mathbf{b}, \mathbf{e}, \mathbf{f}$ indicate the location of the center of the incipient disturbance at $900 \mathrm{hPa}$, and triangles in $\mathbf{e}$ and $\mathbf{f}$ indicate the location of the center of the maximum vorticity in the free atmosphere

6 days starting from 0000 UTC 15 June 2008, approximately 4 days prior to the declaration of Tropical Storm Fengshen by the Joint Typhoon Warning Center (JTWC).

\section{Analysis method}

The $1.0^{\circ}$ gridded (3-hourly) data were created for comparison with the objective analyses. The center of the 
Table 1 Parameter settings of cloud microphysics

\begin{tabular}{|c|c|c|c|c|}
\hline Case & E_mp1 & $\begin{array}{l}\text { E_mp2 } \\
\text { N_mp2 }\end{array}$ & E_mp3 & E_mp4 \\
\hline Psaut_ $\beta_{0}$ & 0.001 & 0.001 & 0.02 & 0.0025 \\
\hline 「_saut & 0.025 & 0.025 & 0.09 & 0.025 \\
\hline 「_sacr & 0.025 & 0.025 & 0.09 & 0.025 \\
\hline cnst_V T_qi $_{\text {T }}$ & - & - & -0.05 & - \\
\hline $\mathrm{cr}$ & 130 & 130 & 130 & 57 \\
\hline CS & 4.84 & 4.84 & 4.84 & 1.0 \\
\hline$C_{-} d$ & 0.6 & 0.6 & 0.6 & 20.0 \\
\hline
\end{tabular}

The parameter Psaut $\beta_{0}$ and $\Gamma$ saut are concerning the autoconversion rate of cloud ice to snow [Eq. (52) and (53) in Tomita 2008], and [_sacr is concerning accretion rate of cloud ice by snow [Eq. (39) in Tomita 2008], cnst_ $V_{T-q i}$ is the constant for terminal velocity of cloud ice $\left(\mathrm{V}_{\mathrm{T}-\mathrm{qi}}\right), \mathrm{cr}$ and $\mathrm{cs}$ are the coefficients of terminal velocity of rain and snow, respectively [Eq. (28) in Tomita 2008], and $C_{-} d$ is concerning the terminal velocity of graupel. In $E_{,} V_{T-q i}$ was formulated as $\mathrm{V}_{\mathrm{T}-\mathrm{qi}}=3.29$ (pqi) $^{0.16}$ [Eq. (5) of Heymsfield and Donner 1990], where $\rho$ is the air density $\left(\mathrm{kg} \mathrm{m}^{-3}\right)$ and qi is cloud ice content $\left(\mathrm{kg} \mathrm{kg}^{-1}\right)$

simulated incipient vortex was identified by the following procedure: (1) the $1.0^{\circ}$ gridded horizontal winds were smoothed twice with a 9-point weighted-mean filter ${ }^{1}$ to calculate relative vorticity, and the resulting relative vorticity was smoothed in the same way; (2) the location of the maximum relative vorticity was detected as the first guess; (3) horizontal winds within a $5.0^{\circ}$ square domain surrounding the first guess were smoothed by operating the 9-point weighted-mean filter 300 times to calculate relative vorticity; and (4) the location of the maximum relative vorticity at the $1-\mathrm{km}$ altitude was defined as the center of the disturbance. ${ }^{2}$ The center of the incipient vortex in the analyses was defined by the maximum location of the 900-hPa relative vorticity. For evaluation of the simulated track and central SLP, the JTWC best track data were used. The $0.25^{\circ}$ gridded Tropical Rainfall Measuring Mission (TRMM) 3B42v7 (Huffman et al. 2007) at 3-h intervals was used to quantify the observed convective activity.

\footnotetext{
${ }^{1}$ This is a horizontal filtering consisting of weighted average of the grid point and the neighboring eight points, with a weight of 1.0 (center), 0.5 (each side), and 0.3 (corner).

2 The $1.0^{\circ}$ gridded center appeared continuous after a 12 -h integration, while the final location of the center was rather discontinuous until the storm attained a certain intensity because of the relatively short lifetime of vorticity peaks that comprised a loosely defined core region of the incipient disturbance. The center of the storm as defined above was located within $\sim 30 \mathrm{~km}$ (a few grid points) of the center defined by a TC tracking method which has been used for general circulation models (e.g., Sugi et al. 2002; Oouchi et al. 2006; Nakano et al. 2015) after the storm satisfied the TC criteria (started deepening). The storm center defined in this study generally agreed better with the circulation center than did that defined by the latter method, presumably being free from the biases that tended to appear when the SLP minimum was used for the definition (Braun 2002).
}

\section{Results and discussion}

\section{Large-scale fields}

Figure 1 presents the large-scale fields in the analyses. On 15 June 2008, the incipient disturbance of Fengshen was located at the eastern edge of the large-scale $(\sim 2000 \mathrm{~km})$ gyre to the north of the equator at $850 \mathrm{hPa}$, where zonal convergence was pronounced (Fig. 1a, b). This is one of the typical types of favorable environment for tropical cyclogenesis (Yoshida and Ishikawa 2013). The precursor of Fengshen (outlined by thick contour lines) moved northwestward with increasing vorticity in the east to northeast of the large-scale gyre (outlined by thin contour lines in Fig. 1c, d). In the vertical, the vorticity maximum was rather limited in the lower troposphere on 16 June, and the distance between the lower and middle tropospheric maxima was $1 \sim 2^{\circ}$ until 19 June (Fig. 1e, f), suggesting that they had different origins. The vertical penetration was slower in the NCEP FNL than in the ECMWF YOTC. In the upper troposphere, a westerly was dominant in the northern hemisphere, indicating a pronounced vertical shear of horizontal wind (Fig. 1c, d).

\section{Track and intensity of Fengshen}

In the macroscopic evolution described above, Fengshen was first detected as tropical depression by JTWC at 0600 UTC on 17 June 2008. The observed and simulated track and central SLP are plotted in Fig. 2. In the best track, the central SLP started decreasing at around 0000 UTC on 18 June (Fig. 2b), and recorded its minimum pressure at 0000 UTC on 21 June. A tendency for a northward track bias was found in the simulations (Fig. 2a), ${ }^{3}$ with earlier onset of pressure reduction in $E_{-}$ $\mathrm{mp} 2$ and $\mathrm{E}_{-} \mathrm{mp} 3$, and an eventual overdevelopment in $\mathrm{E}_{-}$ mp2 (Fig. 2b). In contrast, N_mp2 showed scarce deepening of the initial disturbance. Delayed evolution was found in E_mp4, where the latent heating rate was systematically reduced, especially in the lower troposphere (Table 1; Additional file 1: Figure S1c).

\section{Simulated large-scale fields}

In the following section, we discuss the difference in the simulation results in terms of the origin of the middle tropospheric vorticity disturbance. Figure 3 shows the $400-\mathrm{hPa}$ vorticity fields at 1 -day intervals in the analyses (Fig. 3a-d) and simulations (Fig. 3e-g). The lower tropospheric wind fields in the same period are presented in Additional file 2: Figure S2. To extract largescale disturbances, the $1.0^{\circ}$ gridded horizontal winds were smoothed ten times with a 9-point weighted-mean

\footnotetext{
3 The northward track bias for Fengshen has been commonly seen in many operational forecasts, where it has also been more severe (Cooper and Falvey 2008; Yamada et al. 2016).
} 

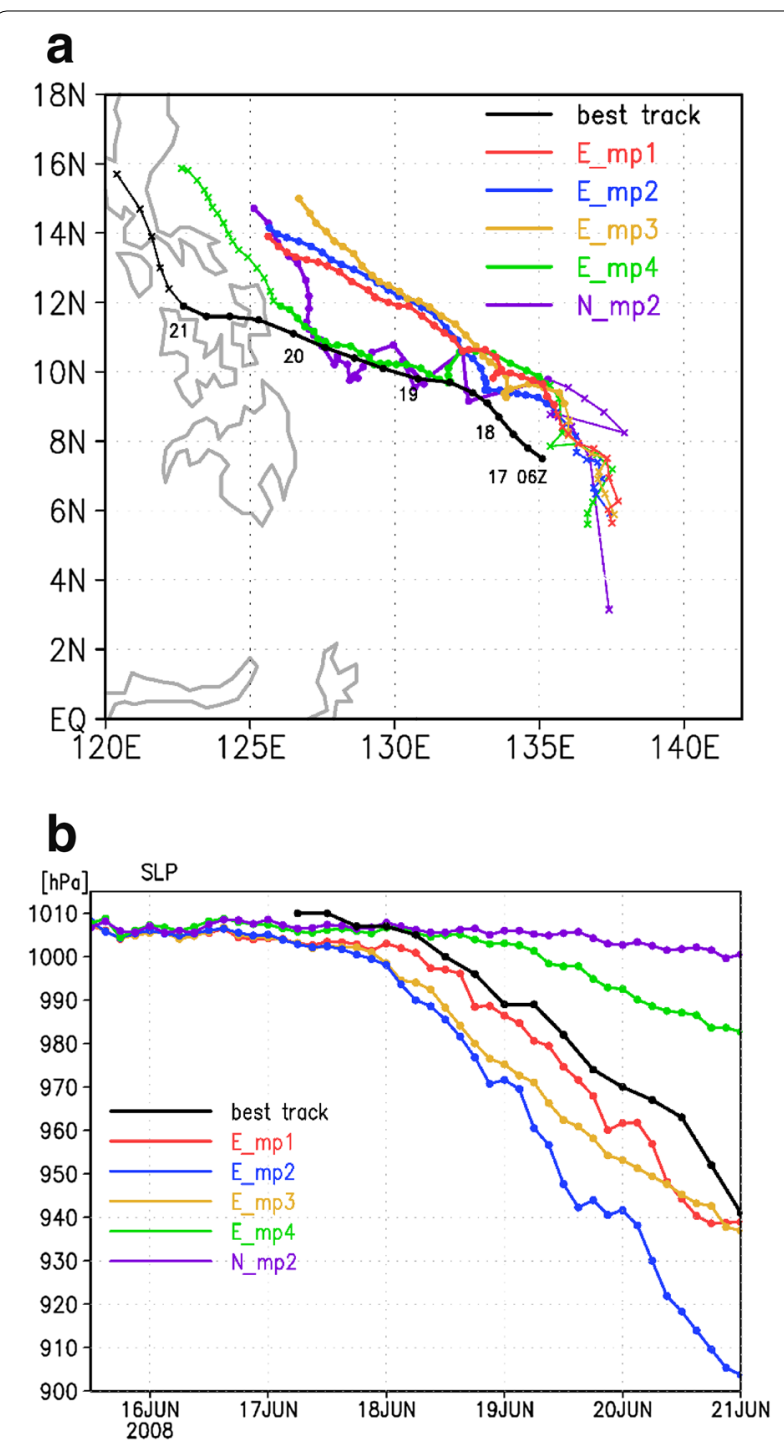

Fig. 2 a Track of Fengshen and $\mathbf{b}$ time series of central sea level pressure (SLP) in the best track (black) and simulations; E_mp1 (red), E_mp2 (blue), E_mp3 (yellow), E_mp4 (green), and N_mp2 (purple). Tracks before 0600 UTC on 17 June and after 0000 UTC on 21 June 2008 are plotted by thin lines and crosses

filter to calculate relative vorticity, and the resulting vorticity was smoothed in the same way. On 15 June 2008 (initial date of simulation), positive vorticity anomalies (troughs) developed around $130^{\circ} \mathrm{E}$ and $150^{\circ} \mathrm{E}$ in the subtropical middle to upper troposphere (Fig. 3a, b), and were extending southeastward and southwestward, respectively. They overlapped the lower tropospheric gyre. In the subsequent three days, the middle tropospheric trough intensified and migrated northwestward with a vorticity maximum at $130^{\circ}-140^{\circ} \mathrm{E}$ (Fig. 3c, d). The center of the low-level incipient vortex (crosses in Fig. 3) was located on the northeastern rim of the vorticity maximum during the migration. Prior to the intensification of the middle tropospheric vorticity, convective activity was enhanced in the broad domain corresponding to the lower tropospheric large-scale gyre (shading in Fig. 3; Additional file 2: Figure S2). The growth of middle tropospheric trough was slower in NCEP FNL than in the ECMWF YOTC (Fig. 3c, d).

The evolutions described above were reasonably simulated in E_mp2 (Fig. 3e; Additional file 2: Figure S2c), E_mp1, and E_mp3 (not shown). In N_mp2, the results were substantially different, with weak development of the middle tropospheric trough, which was less coherent to the latent heat release and lower tropospheric gyre (Fig. 3f; Additional file 2: Figure S2d). In E_mp4, a weak trough was formed after 16 June, with smaller latent heat release over the lower tropospheric gyre than in E_mp2 (Fig. 3g; Additional file 2: Figure S2e). These results indicate that the large-scale latent release associated with the lower tropospheric conditions (e.g., the active period of BSISO) was important to the growth of the middle tropospheric trough and subsequent development of TC within the vertically coherent large-scale positive vorticity anomaly. In the lower troposphere, a wave train-like structure was confirmed in the analyses and the TC developing simulation, with northeast-southwest phase tilt in parallel to the middle tropospheric trough (Additional file 2: Figure $\mathrm{S} 2 \mathrm{a}-\mathrm{c}$ ). The structure was clearer in $\mathrm{E} \_\mathrm{mp} 2$ than in the analyses, while it was less evident in E_mp4 and N_mp2.

In order to interpret the differences in evolution among the simulations (Fig. $4 \mathrm{e}-\mathrm{g}$ ), the static stability $N^{2}=g / \theta_{0} \partial \theta / \partial z$ and intertial stability $I^{2}=(f+$ $\left.\partial r \nu_{\mathrm{T}} / r \partial r\right)\left(f+2 \nu_{\mathrm{T}} / r\right)$ were examined, where $\theta$ is potential temperature, $\theta_{0}(=300 \mathrm{~K})$ is the reference potential temperature, $r$ is radius, $g\left(=9.8 \mathrm{~m} \mathrm{~s}^{-2}\right)$ is the gravitational acceleration, $f$ is the Coriolis parameter, and $v_{\mathrm{T}}$ is the tangential wind. According to the Eliassen's (1952) balanced model, $N^{2}$ and $I^{2}$ have been used to diagnose the vertical and horizontal extents of the thermally forced transverse circulation, respectively (Schubert and Hack 1982; Shapiro and Willoughby 1982). Figure 4 shows the $N^{2}, I^{2}$, and $\Gamma=\left(N^{2} / I^{2}\right)^{1 / 2}$, averaged within the $600-\mathrm{km}$ radius of the large-scale vortex in the simulations. ${ }^{4}$

On 15 June, $I^{2}$ was small above the $6-\mathrm{km}$ altitude, which led to a large $\Gamma$ in both analyses (Fig. $4 a-c)$. On 17 June, convective activity induced thermal stabilization and enhancement of the vortex (Fig. $4 \mathrm{~d}, \mathrm{e}, \mathrm{g}$ ). In N_mp2 (E_mp2 and E_mp4), the increase in $I^{2}$ was limited below (extended above) the $6-\mathrm{km}$ altitude, reflecting

\footnotetext{
${ }^{4}$ The center of the large-scale vortex was defined by the maximum location of the smoothed $1-\mathrm{km}$ altitude vorticity. The 9-point weighted-mean filtering was operated 20 times for the 1.0-degree gridded data to obtain the smoothed fields.
} 


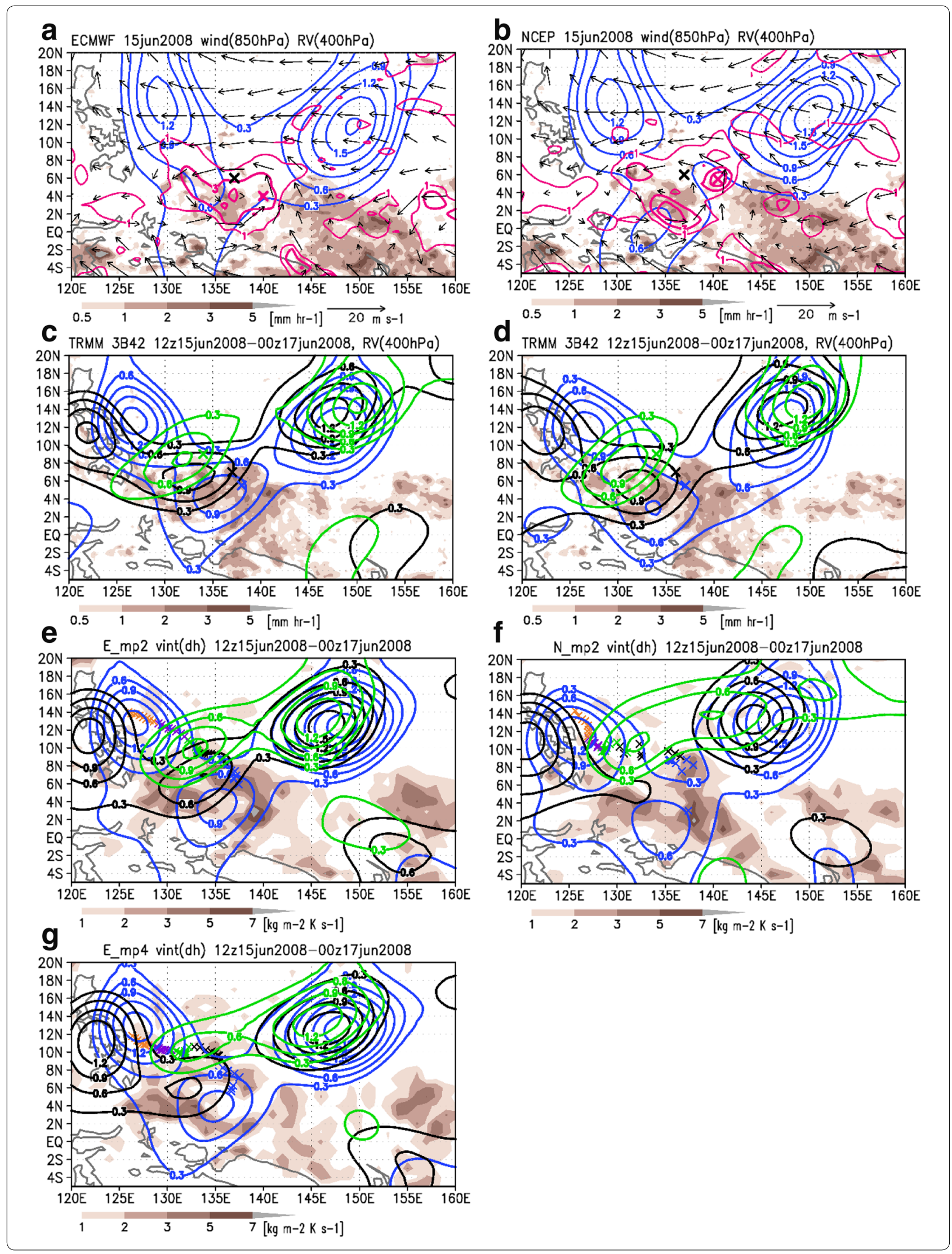


(See figure on previous page.)

Fig. 3 Horizontally smoothed 400-hPa relative vorticity (blue), 850-hPa wind vectors, and relative vorticity (red) at 0000 UTC on 15 June 2008 , in the $\mathbf{a}$ ECMWF YOTC and $\mathbf{b}$ NCEP FNL, with the precipitation rate averaged between 1200 UTC on 13 June and 0000 UTC on 15 June 2008, in TRMM 3B42v7 (shading). Horizontally smoothed 400-hPa relative vorticity at 0000 UTC on 16 (blue), 17 (black), and 18 (green) June 2008, in the c ECMWF YOTC and d NCEP FNL with the precipitation rate averaged between 1200 UTC on 15 June and 0000 UTC on 17 June 2008 in TRMM 3B42v7 (shading). Same as c $\mathbf{d}$ d, except for the simulation case $\mathbf{e}$ E_mp2, $\mathbf{f} \mathrm{N} \_\mathrm{mp} 2$, and $\mathbf{g}$ E_mp4 at the 7.6-km altitude (approximately corresponding to $400 \mathrm{hPa}$ ), with the vertically integrated latent heating rate (shading). In all panels, the unit of relative vorticity is $10^{-5} \mathrm{~s}^{-1}$ and only positive values are shown. Crosses indicate the center of the incipient disturbance on 15 (red), 16 (blue), 17 (black), 18 (green), 19 (purple), and 20 (orange) June 2008. Black crosses in $\mathbf{a}$ and $\mathbf{b}$ indicate the center of the large-scale gyre

the different strengths of diabatic forcing. As a result, $\Gamma$ in the middle troposphere was smaller in E_mp2 and E_mp4 than in N_mp2 (Fig. 4f), and the Rossby depth $(=1 /(\Gamma k) ; k$ is the radial wave number), which represents the vertical size of the forced circulation (Schubert and McNoldy 2010), in E_mp2 was approximately 1.6 times that in N_mp2. This elucidates the subsequent coupling of the large-scale lower and middle tropospheric vortices in E_mp2, which was substantially weaker in N_mp2.

\section{Simulated evolution of the TC inner core}

Given the spin-up of the large-scale circulation in both the lower and the middle troposphere loosely coupled by enhanced convection, what determines the onset of the pressure deepening and subsequent intensification? Figure 5 presents the time evolution of the inner core of the simulated storm. Because of the ambiguous structure in N_mp2, only the averages within the 50- and 100-km radius are presented for this case (Fig. 5e).

Deep latent heating associated with convective events within the $50-\mathrm{km}$ radius occurred at $12 \mathrm{~h}$ or at shorter intervals during 16-17 June in the TC developing cases (Fig. $5 \mathrm{a}-\mathrm{c}$ ). An increase in the azimuthal mean tangential wind in the middle troposphere occurred simultaneously or several hours after the deep convective events (upper panels). An increase in the tangential wind was less clear in E_mp4 and N_mp2 in this period (Fig. 5d, e). This difference was attributable to the weakness of the largescale vorticity that preconditioned the intensification of the TC inner core in these runs. In the TC developing runs, an abrupt increase in the column-integrated latent heat release occurred over a 1 1.5-day period around 18 June (Fig. $5 \mathrm{a}-\mathrm{c}, \mathrm{f}$ ). As a measure of the vertical tilt of the inner core, the distance between the locations of the vorticity maximum at the 1 - and $7.6-\mathrm{km}$ altitudes (approximately corresponding to 900 and $400 \mathrm{hPa}$, respectively) was calculated (black lines in the lower panels). Concurrent with the outbreak of the latent heat release, the distance sharply decreased, and the acceleration rate of the middle tropospheric tangential wind (purple lines in the lower panels) abruptly rose. This indicates the establishment of an upright deep TC inner core within the southwestward slanted large-scale vorticity fields (Fig. 3). In E_mp2 and E_mp3, the convective enhancement was more vigorous and was rooted below the $2-\mathrm{km}$ altitude, where it was sustained for $\sim 10 \mathrm{~h}$ (Fig. 5b, c, f) around 0000 UTC on 18 June. These elucidate the earlier onset of pressure deepening in these runs (Fig. 2b). In E_mp1 and E_mp2, a prominent increase in latent heat release was confined very close to the storm center $(\mathrm{r}<22 \mathrm{~km})$ during the rapid change (late on 18 June; Fig. 5a, b). In these runs, a very strong single convective event made a substantial contribution to the formation of the TC inner core. Figure 6 presents zoomed plots of the relative vorticity before (Fig. 6a, c) and during the explosive latent heat release (Fig. 6b, d) in E_mp2. Before the event, the lowlevel incipient vortex consisted of small vorticity peaks, with relatively short lifetimes. The flow fields in the middle troposphere were elongated in the southwestward direction, and the centers of both two levels did not coincide. In Fig. 6b, d, a long-lasting deep convective event can be seen close to the center, and a vertically coherent vorticity peak, or a TC inner core, was established in this region. Not all the deep convective events within the $22-\mathrm{km}$ radius led to significant intensification of axisymmetric tangential wind (Fig. 5). In some cases, the merger of the small vorticity peaks accounted for the development of a TC inner core, rather than the occurrence of a few prominent convective events (e.g., E_mp4). In summary, the deep explosive convective event, analogous to a "hot tower," does not appear to be a necessary nor sufficient condition for the establishment of a TC inner core or the onset of pressure reduction. However, it certainly played a crucial role when it occurred with the right timing and location in association with an environment that was preconditioned by the superposition of multiple (dynamical and convective) large-scale factors with different origins.

\section{Conclusions}

Large-scale fields and inner-core processes relevant to the formation and intensification of Typhoon Fengshen (2008) were investigated by a set of global cloud-permitting (3.5-km mesh) simulations. Fengshen was formed in 

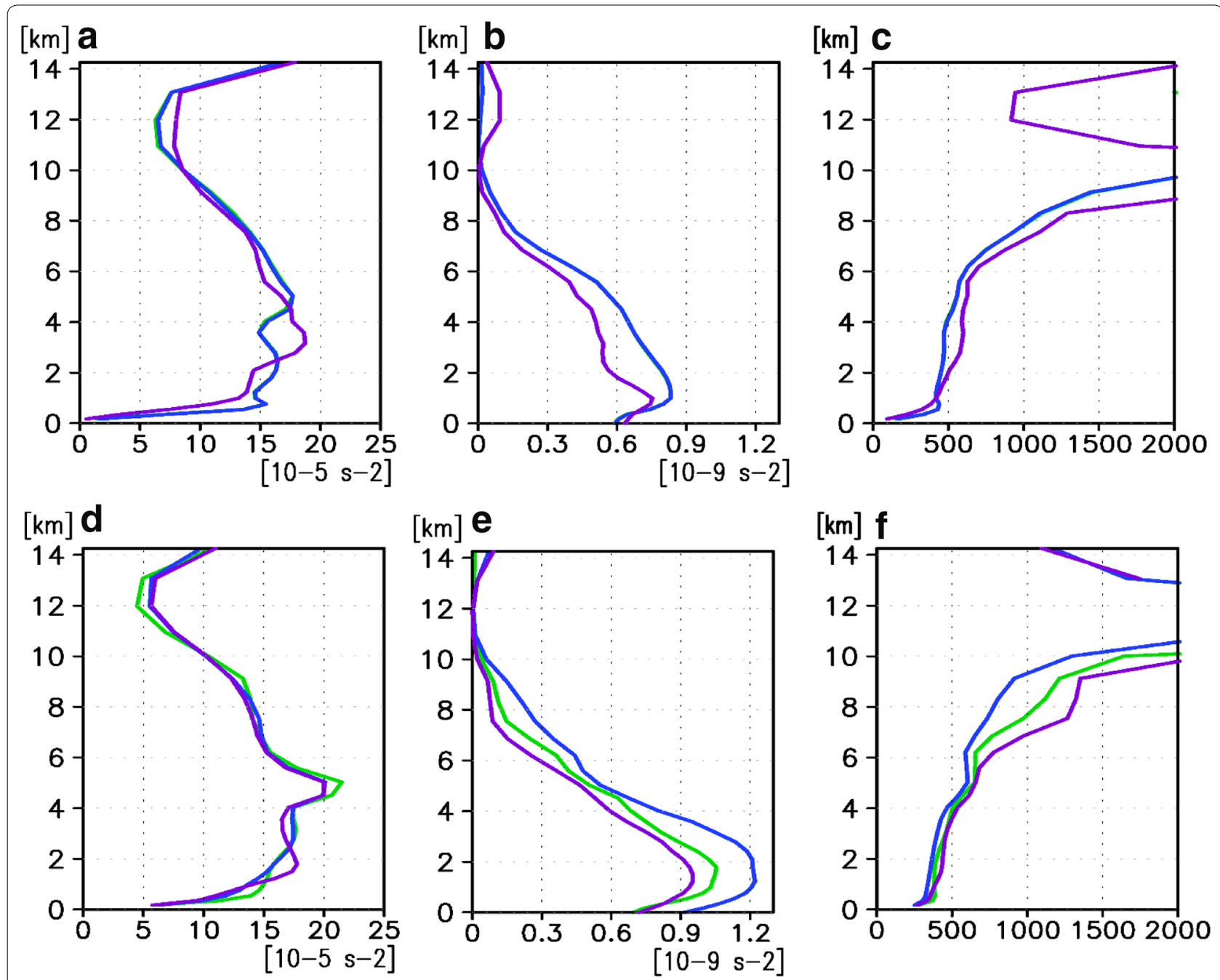

$[\mathrm{km}] \mathbf{f}$

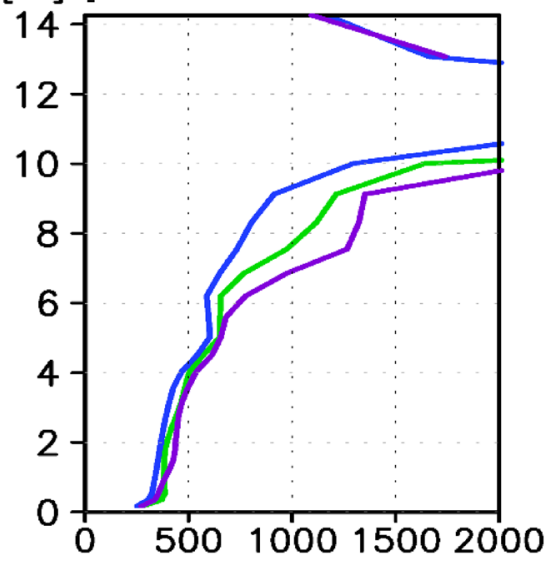

$[\mathrm{km}] \mathbf{9}$

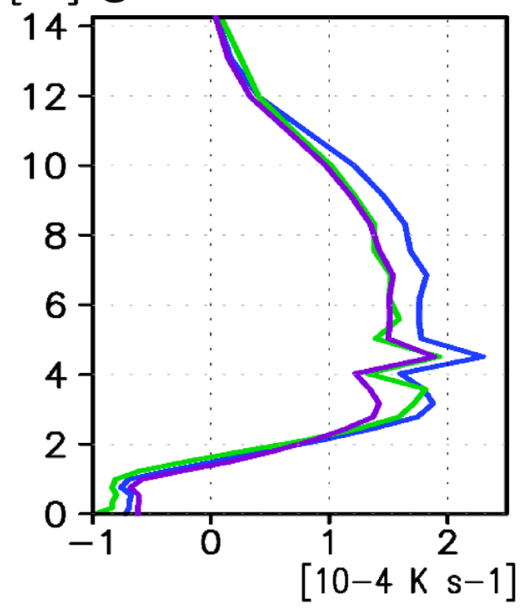

Fig. 4 Vertical profiles of $\mathbf{a}$, $\mathbf{d}$ ) static stability, $\mathbf{b}$, e inertial stability, $\mathbf{C}, \mathbf{f} \Gamma$, and $\mathbf{g}$ latent heating rate averaged within the 600-km radius of the largescale lower tropospheric vortex $\mathbf{a}-\mathbf{c}$ at 0003 UTC on 15 June, $\mathbf{d}-\mathbf{f}$ at 0000 UTC on 17 June, and $\mathbf{g}$ the average between 1800 UTC 15 June and 0000 UTC 17 June 2008, in E_mp2 (blue), E_mp4 (green), and N_mp2 (purple) 


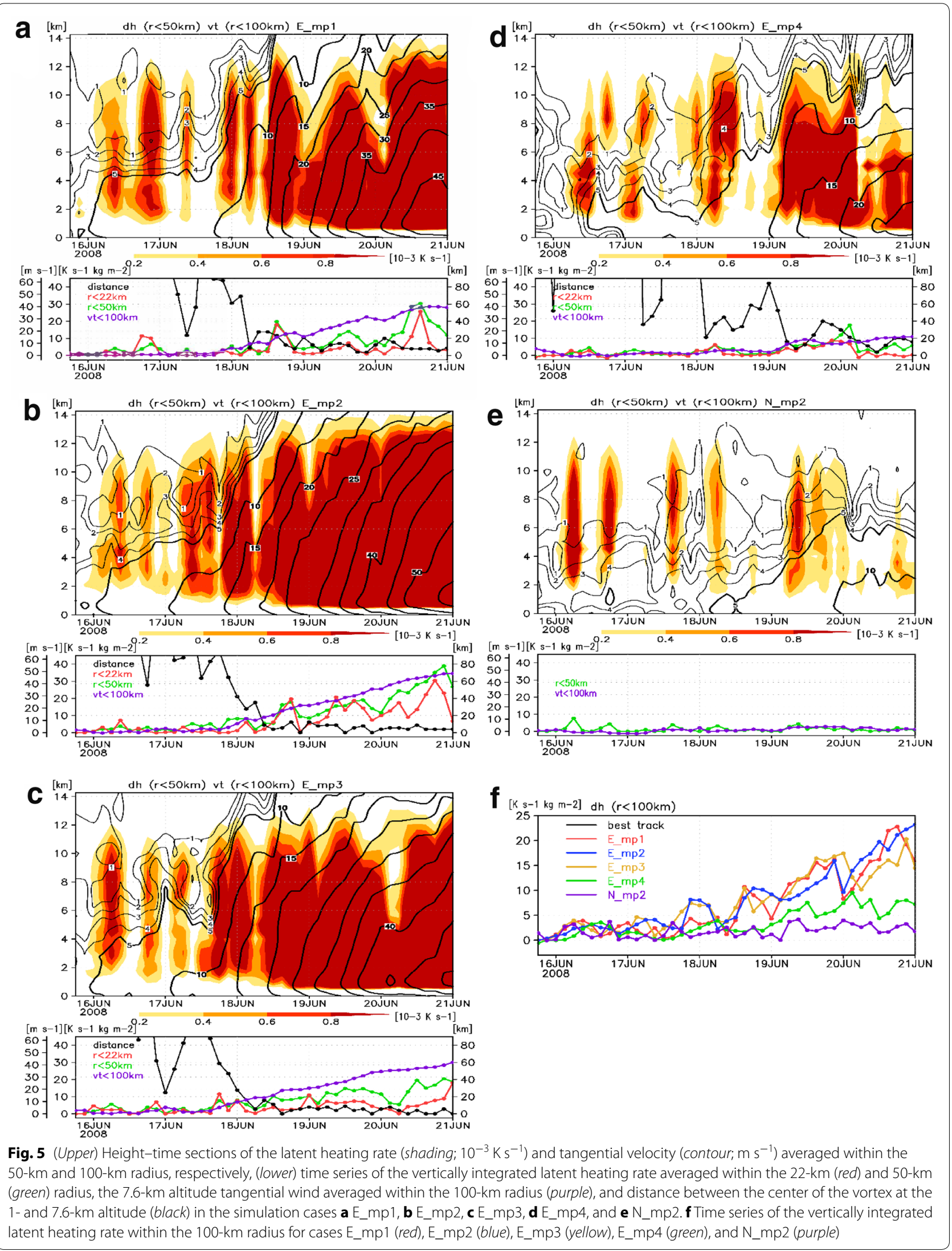




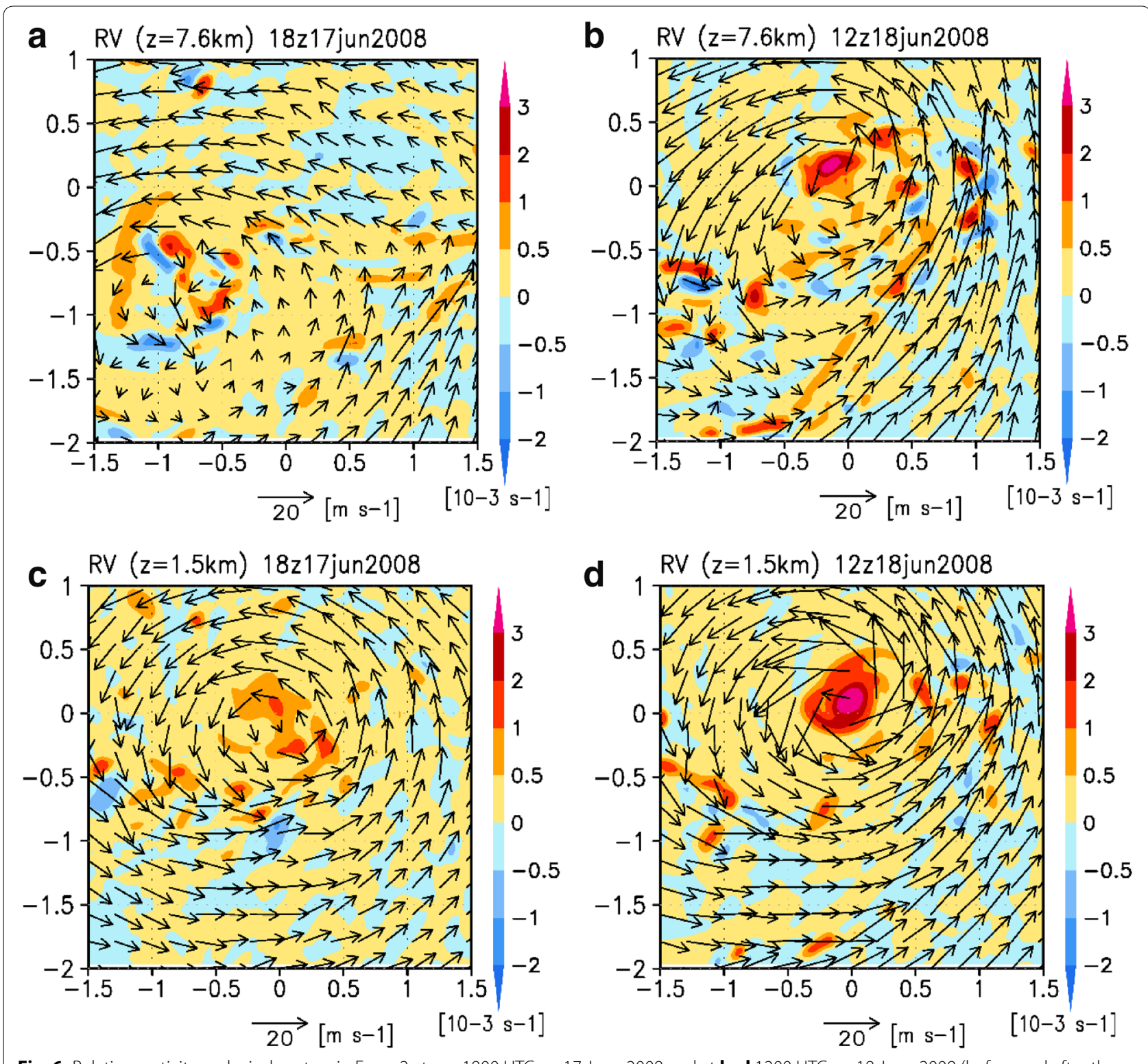

Fig. 6 Relative vorticity and wind vectors in E_mp2 at a, c 1800 UTC on 17 June 2008 and at b, d 1200 UTC on 18 June 2008 (before and after the onset of intensification) at the $\mathbf{a}, \mathbf{b} 7.6-\mathrm{km}$ and $\mathbf{c}, \mathbf{d} 1.5-\mathrm{km}$ altitude in the coordinates with the origin located at the storm center

mid-June during the onset of the WNP monsoon and the active phase of a BSISO event.

The TC genesis was preceded by the formation of a lower tropospheric large-scale gyre associated with enhanced equatorial westerlies and a middle tropospheric wave trough intruding above the gyre in both the ECMWF YOTC and the NCEP FNL. The incipient disturbance of Fengshen was located on the northeastern rim of the large-scale gyre, where moisture convergence was pronounced. Differences between the analysis datasets were found in the development of the trough and the horizontal distribution of moisture.
To understand the impact of atmospheric conditions and the convective effects on the TC environment and precursor disturbance, five simulations were performed by varying the initial data (ECMWF YOTC, NCEP FNL) and cloud microphysics settings (mp1-4).

In three simulations initialized using the ECMWF YOTC, a middle tropospheric trough developed over a few days following a large-scale latent heat release above the lower tropospheric gyre, allowing for the successive occurrence of deep convective events within the $50-\mathrm{km}$ radius of the incipient disturbance, and a deep upright $\mathrm{TC}$ inner core was established. 
In the run with reduced latent heating (mp4), the trough was weak and TC formation was significantly delayed, suggesting the importance of the latent heat release over the lower tropospheric gyre to the intensification of the middle tropospheric trough and vertical coupling of the large-scale vorticity, which facilitated the acceleration of middle tropospheric tangential wind around the incipient low-level vortex $(<50-100 \mathrm{~km})$.

In the run initialized using the NCEP FNL, where the latent heat release averaged over the entire simulation was the same as its ECMWF YOTC counterpart, neither the trough nor a TC developed, lacking the collocation between the latent heat release and large-scale gyre in the early period. Under the vertical wind shear increasing poleward, it was critical for the incipient vortex to experience upward penetration before it moved farther northward.

The wave train (SWT) is a dominant unstable mode under the WNP summertime background state (Aiyyer and Molinari 2003; Kuo et al. 2001; Lau and Lau 1990; Li 2006) and easily manifests from various disturbances (Xu et al. 2014; Wu and Duan 2015). In the case of Fengshen, our results suggest that the superposition of large-scale disturbances with different origins, namely a middle tropospheric trough and lower tropospheric gyre associated with the active period of a BSISO event, formed the wave train. The earlier and stronger development of a wave train-like structure down to the lower troposphere in the TC developing simulations was considered to be responsible for the northward displacement, as well as the tendency toward over-intensification.

It is a very challenging task to simulate/predict the behavior of multiple disturbances that may excite the wave train accurately, but that will improve the predictability of TC genesis in the WNP. A high-resolution global model may serve as a useful tool to achieve this.

\section{Additional files}

Additional file 1: Figure S1. Vertical profiles of $\mathbf{a}$ temperature and $\mathbf{b}$ water vapor content differences (E_mp2 minus N_mp2) averaged for the initial three hours, and $\mathbf{c}$ latent heating rate in E_mp2 (blue), E_mp4 (green), and N_mp2 (purple) averaged for the 6-day integration (the difference from E_mp2 is also plotted by thin lines), over the $\left(120^{\circ}-160^{\circ} \mathrm{E}\right.$, $10^{\circ} \mathrm{S}-20^{\circ} \mathrm{N}$ ) domain.

Additional file 2: Figure S2. Relative vorticity (shading) and wind vectors at 0000 UTC on 17 June 2008, in the a ECMWF YOTC and b NCEP FNL at $850 \mathrm{hPa}$, and those in the simulation cases $\mathbf{c}$ E_mp2, d N_mp2, and e E_mp4 at the 1.5-km altitude. Contour lines for the relative vorticity averaged between 6-h before and after 0000 UTC on 16 (blue) and 18 (green) June 2008, at the same height level $\left(1,5\right.$, and $\left.10 \times 10^{-5} \mathrm{~s}^{-1}\right)$ are also plotted. Crosses in $\mathbf{e}-\mathbf{f}$ indicate the center of the large-scale gyre.

\section{Abbreviations}

ECMWF: European Center for Medium-range Weather Forecasting; JTWC: Joint Typhoon Warning Center; MSTRN: model simulation radiation Transfer code; NCEP: National Centers for Environmental Prediction; NICAM: Nonhydrostatic Icosahedral Atmospheric Model; NOAA: National Oceanic and Atmospheric Administraion; SLP: sea level pressure; TCG: tropical cyclogenesis; TRMM: tropical rainfall measuring mission; YOTC: year of tropical convection.

\section{Authors' contributions}

TN conducted numerical simulation and analysis, and drafted the manuscript; HY selected the case of Fengshen (2008) and provided insight into this TCG case based on analysis of observation and simulation data; MN helped with the TC tracking analysis and drafting the manuscript; HK provides insight into the tropical cyclogenesis over the Philippine Sea; MS conducted reference numerical experiments using the Japan Meteorological Agency Nonhydrostatic model (JMANHM); and RY provided the analysis results based on Yoshida and Ishikawa's (2013) method for Typhoon Fengshen. All the authors joined the discussion on the results, and read and approved the manuscript.

\section{Author details}

1 Japan Agency for Marine-Earth Science and Technology, 3173-25 Showa-machi, Kanazawa-ku, Yokohama, Kanagawa 236-0001, Japan. ${ }^{2}$ Faculty of Science, University of the Ryukyus, Nishihara, Japan. ${ }^{3}$ Atmosphere and Ocean Research Institute, The University of Tokyo, Kashiwa, Japan. ${ }^{4}$ Meteorological Research Institute, Tsukuba, Japan. ${ }^{5}$ Advanced Institute for Computational Science, RIKEN, Kobe, Japan.

\section{Acknowledgements}

The authors express their thanks to Drs. Yoshiaki Miyamoto and Kozo Nakamura for valuable discussions. They appreciate a number of insightful comments from two anonymous reviewers. The data providers are also acknowledged. The NOAA_OI_SST_V2 data were provided by the NOAA/ OAR/ESRL PSD, Boulder, Colorado, USA, from their Web site at http://www. esrl.noaa.gov/psd/. The TRMM 3B42v7 was provided by National Aeronautics and Space Administration (NASA) and Japan Aerospace Exploration Agency (JAXA). The ECMWF YOTC operational analysis was provided by ECMWF from their Web site at http://apps.ecmwf.int/datasets/data/yotc-od/levtype=sfc/ type $=a n /$. The NCEP final analysis was provided by NCEP and downloaded from the Research Data Archive (http://rda.ucar.edu/datasets/ds083.2/) which is managed by the Data Support Section of the Computational and Information Systems Laboratory at the National Center for Atmospheric Research in Boulder, Colorado. The best track data were provided by JTWC form their Web site at http://www.usno.navy.mil/NOOC/nmfc-ph/RSS/jtwc/best_tracks/. All the simulations were conducted on the Earth Simulator at JAMSTEC. TN is supported by JSPS KAKENHI Grant Number JP26400475. MN is supported by HPCI Strategic Programs for Innovative Research Field 3 of MEXT and FLAGSHIP 2020 project of the Ministry of Education, Culture, Sports, Science, and Technology (MEXT).

\section{Competing interests}

The authors declare that they have no competing interests.

Received: 7 March 2016 Accepted: 25 November 2016 Published online: 01 December 2016

\section{References}

Aiyyer AR, Molinari J (2003) Evolution of mixed Rossby-gravity waves in idealized MJO environments. J Atmos Sci 60:2837-2855

Bister M, Emanuel KA (1997) The genesis of Hurricane Guillermo: TEXMEX analyses and a modeling study. Mon Wea Rev 125:2662-2682

Braun SA (2002) A cloud-resolving simulation of Hurricane Bob (1991): storm structure and eyewall buoyancy. Mon Wea Rev 130:1573-1592

Briegel LM, Frank WM (1997) Large-scale influences on tropical cyclogenesis in the western north Pacific. Mon Wea Rev 125:1397-1413

Cooper GA, Falvey RJ (2008) 2008 Annual Tropical Cyclone Report. US Naval Maritime Forecast Center/Joint Typhoon Warning Center Pearl Harbor, Hawaii 
Dickinson M, Molinari J (2002) Mixed Rossby-gravity waves and western Pacific tropical cyclogeneesis. Part I: synoptic evolution. J Atoms Sci 59:2183-2196

Eliassen A (1952) Slow thermally or frictionally controlled meridional circulation in a circular vortex. Astrophysica Norvegica 5:19-60

Frank WM, Roundy PM (2006) The role of tropical waves in tropical cyclogenesis. Mon Wea Rev 134:2397-2417

Fu B, Li T, Peng MS, Weng F (2007) Analysis of tropical cyclogenesis in the western north pacific for 2000 and 2001. Weather Forecast 22:763-780

Geng B, Yoneyama K, Shirooka R, Yoshizaki M (2011) Characteristics of precipitation systems and their environment during the onset of the western north Pacific summer monsoon in 2008. J Meteor Soc Japan 89A:1-25

Hashino T, Satoh M, Hagihara Y, Kubota T, Matsui T, Nasuno T, Okamoto H (2013) Evaluating cloud microphysics from NICAM against CloudSat and CALIPSO. J Geophys Res Atmos. doi:10.1002/jgrd.50564

Hashino T, Satoh M, Hagihara Y, Kato S, Kubota T, Matsui T, Nasuno T, Okamoto $\mathrm{H}$, Sekiguchi M (2016) Evaluating Arctic cloud radiative effects simulated by NICAM with A-train. J Geophys Res Atmos. doi:10.1002/2016JD024775

Hendricks EA, Montgomery MT, Davis CA (2004) The role of vortical hot towers in the formation of Tropical Cyclone Diana (1984). J Atmos Sci 61:1209-1232

Heta Y (1990) An analysis of tropcial wind fields in relation to Typhoon formation over the western Pacific. J Meteor Soc Jpn 68:65-77

Heymsfield AJ, Donner $\sqcup$ (1990) A scheme for parameterizing ice cloud water content in general circulation models. J Atmos Sci 47:1865-1877

Holland GJ (1995) Scale interaction in the western Pacific monsoon. Meteorol Atmos Phys 56:57-79

Houze RA Jr (2010) Clouds in tropical cyclones. Mon Wea Rev 138:293-344

Hsu P-C, Li T, Tsou C-H (2011) Interactions between boreal summer intraseasonal oscillations and synoptic-scale disturbances over the western North Pacific. Part I: energetics diagnosis. J Climate 24:927-941

Huffman GJ, Adler RF, Bolvin DT, Gu G, Nelkin EJ, Bowman KP, Hong Y, Stocker EF, Wolff DB (2007) The TRMM multi-satellite precipitation analysis: Quasiglobal, multi-year, combined-sensor precipitation estimates at fine scale. J Hydrometeor 8:38-55

Katsumata M, Yamada H, Kubota H, Moteki Q, Shirooka R (2013) Observed evolution of northward-propagating intra seasonal variation over the western Pacific: a case study in boreal early summer. Mon Wea Rev 141:690-706

Kerns BW, Chen SS (2013) Cloud clusters and tropical cyclogenesis: developing and nondeveloping systems and their large-scale environment. Mon Wea Rev 141:192-210

Kodama C, Yamada Y, Noda A, Kikuchi K, Kajikawa Y, Nasuno T, Tomita T, Yamaura T, Takahashi H, Hara M, Kawatani Y, Satoh M, Sugi M (2015) A 20-year climatology of a NICAM AMIP-type simulation. J Meteorol Soc Jpn 93:393-424

Kuo H-C, Chen JH, Williams RT, Chang C-P (2001) Rossby waves in zonally opposing mean flow: behavior in northwest Pacific. J Atmos Sci 58:1035-1050

Lau KH, Lau NC (1990) Observed structure and propagation characteristics of tropical summertime synoptic scale disturbances. Mon Wea Rev 118:1888-1913

LiT (2006) Origin of the summer time synoptic-scale wave train in the Western North Pacific. J Atmos Sci 63:1093-1102

Liebmann B, Hendon HH, Glick JD (1994) The relationship between tropical cyclones of the western Pacific and Indian Oceans and the MaddenJulian oscillation. J Meteor Soc Jpn 72:401-412

Madden R, Julian P (1971) Detection of a 40-50 day oscillation in the zonal wind in the tropical Pacific. J Atmos Sci 28:702-708

Maloney ED, Hartmann DL (2001) The Madden-Julian oscillation, barotropic dynamics, and North Pacific tropical cyclone formation. Part I: observations. J Atmos Sci 58:2545-2558

Mellor GL, Yamada T (1982) Development of a turbulent closure model for geophysical fluid problems. Rev Geophys Space Phys 20:851-875

Miura H, Suematsu T, Nasuno T (2015) An ensemble hindcast of the MaddenJulian oscillation during the CINDY2011/DYNAMO field campaign and influence of seasonal variation of sea surface temperature. J Meteor Soc Jpn 93A:115-137

Nakanishi M, Niino H (2006) An improved Mellor-Yamada level-3 model: its numerical stability and application to a regional prediction of advection fog. Bound Layer Meteorol 119:397-407
Nakano M, Sawada M, Nasuno T, Satoh M (2015) Intraseasonal variability and tropical cyclogenesis in the western North Pacific simulated by a global nonhydrostatic atmospheric model. Geophys Res Lett. doi:10.1002/201 4GL062479

Nasuno T (2013) Forecast skill of Madden-Julian oscillation events in a global nonhydrostatic model during the CINDY2011/DYNAMO observation period. Sci Online Lett Atmos 9:69-73

Noda A, Oouchi K, Satoh M, Tomita H, Iga S, Tsushima Y (2010) Importance of the subgrid-scale turbulent moist process: cloud distribution in global cloud-resolving simulations. Atmos Res 96:208-217

Nolan DS (2007) What is the trigger for tropical cyclogenesis? Aust Meteor Mag 56:241-266

Oouchi K, Yoshimura J, Yoshimura H, Mizuta R, Kusunoki S, Noda A (2006) Tropical cyclone climatology in a global warming climate as simulated in a 20-km-mesh global atmospheric model: frequency and intensity analysis. J Meteorol Soc Jpn 84:259-276. doi:10.2151/jmsj.84.259

Reynolds RW, Rayner NA, Smith TM, Stokes DC, Wang W (2002) An improved in situ and satellite SST analysis for climate. J Clim 15:1609-1625

Ritchie EA, Holland GJ (1997) Scale interactions during the formation of typhoon Irving. Mon Wea Rev 125:1377-1396

Ritchie EA, Holland GJ (1999) Large-scale patterns associated with tropical cyclogenesis in the western Pacific. Mon Wea Rev 127:2027-2043

Sadler JS (1976) A role of the tropical upper tropospheric trough in early season typhoon development. Mon Weather Rev 104:1266-1278

Satoh M, Matsuno T, Tomita H, Miura H, Nasuno T, Iga S (2008) Nonhydrostatic Icosahedral Atmospheric Model (NICAM) for global cloud-resolving simulations. J Comput Phys 227:3486-3514. doi:10.1016/j.jcp.2007.02.006

Satoh M, Tomita H, Yashiro H, Miura H, Kodama C, Seiki T, Noda A, Yamada Y, Goto D, Sawada M, Miyoshi T, Niwa Y, Hara M, Ohno Y, Iga S, Arakawa T, Inoue T, Kubokawa H (2014) The Non-hydrostatic Icosahedral atmospheric model: description and development. Prog Earth Planet Sci. doi:10.1186/s40645-014-0018-1

Schreck CJ, Molinari J (2011) Tropical cyclogenesis associated with Kelvin waves and Madden-Julian oscillation. Mon Weather Rev 139:2723-2734

Schreck CJ, Molinari J, Mohr KI (2011) Attributing tropical cyclogenesis to equatorial waves in the western North Pacific. J Atmos Sci 68:195-209

Schubert WH, Hack JJ (1982) Inertial stability and tropical cyclone development. J Atmos Sci 39:1687-1697

Schubert WH, McNoldy BD (2010) Application of the concepts of Rossby length and Rossby depth to tropical cyclone dynamics. J Adv Model Earth Syst 2, Art \#7: 13 pp

Sekiguchi M, Nakajima T (2008) A k-distribution-based radiation code and its computational optimization for an atmospheric general circulation model. J. Quant Spectrosc Radiat Trans 109:2779-2793

Shapiro LJ, Willoughby HE (1982) The response of balanced hurricanes to local sources of heat and momentum. J Atmos Sci 39:378-394

Simpson J, Halverson JB, Ferrier BS, Petersen WA, Simpson RH, Blakeslee R, Durden SL (1998) On the role of "hot towers" in tropical cyclone formation. Meteor Atmos Phys 67:15-35

Sugi M, Noda A, Sato N (2002) Influence of global warming on tropical cyclone climatology: an experiment with JMA global climate model. J Meteorol Soc Jpn 80:249-272. doi:10.2151/jmsj.80.249

Tam C-Y, Li T (2006) The origin and dispersion characteristics of the observed tropical summertime synoptic-scale waves over the western Pacific. Mon Weather Rev 134:1630-1646

Tomita H (2008) New micorphysics with five and six categories with diagnostic generation of cloud ice. J Meteor Soc Jpn 86A:121-142

Waliser DE, Moncrieff MW et al (2012) The "year" of tropical convection (May 2008-April 2010): climate variability and weather highlights. Bull Am Meteor Soc 93:1189-1218. doi:10.1175/2011BAMS3095.1

Wang B, Rui H (1990) Synoptic climatology of transient tropical intra seasonal convection anomalies: 1975-1985. Meteorol Atmos Phys 44:43-61

Warner TT, Peterson RA, Treadon RE (1997) A tutorial on lateral boundary conditions as a basic and potentially serious limitation to regional numerical weather prediction. Bull Am Meteorol Soc 78:2599-2617

Wu L, Duan J (2015) Extended simulation of tropical cyclone formation in the western North Pacific monsoon trough. J Atmos Sci 72:4469-4485

Xu Y, Li T, Peng M (2013) Tropical cyclogenesis in the western North Pacific as revealed by the 2008-2009 YOTC data. Weather Forecast 28:1038-1056. doi:10.1175/WAF-D-12-00104.1 
Xu Y, LiT, Peng M (2014) Roles of synoptic-scale wave train, intra seasonal oscillation, and high-frequency eddies in genesis of Typhoon Manyi (2001). J Atmos Sci 71:3706-3722. doi:10.1175/JAS-D-13-0406.1

Yamada H, Nasuno T, Yanase W, Shirooka R, Satoh M (2012) Genesis of Typhoon Fengshen (2008) from Vortex Superposition: PALAU Field Experiment and a Global Cloud-Resolving Simulation. In: Abstracts of the 30th conference on Hurricane and Tropical Meteorology, Ponte Vedra Beach, Florida, 15-20 April 2012

Yamada H, Nasuno T, Yanase W, Satoh M (2016) Role of the vertical structure of a simulated tropical cyclone in its motion: a case study of Typhoon Fengshen (2008). Sci Online Lett Atmos 12:203-208. doi:10.2151/ sola.2016-041

Yamasaki M (1983) A furtuer study of the tropical cyclone without parameterizing the effects of cumulus convection. Papers Meteorol Geophys 34:221-260
Yanai M (1961) A detailed analysis of typhoon formation. J Meteorol Soc Jpn 39:187-214

Yoshida R, Ishikawa H (2013) Environmental factors contributing to tropical cyclone genesis over the western North Pacific. Mon Weather Rev 141:451-467

Yoshida R, Kajikawa Y, Ishikawa H (2014) Impact of boreal summer intraseasonal oscillation on environment of tropical cyclone genesis over the western North Pacific. Sci Online Lett Atmos 10:15-18. doi:10.2151/ sola.2014-004

Zhou X, Wang B (2007) Transition from an eastern Pacific upper-level mixed Rossby-gravity wave to a western Pacific tropical cyclone. Geophys Res Lett 34:L24801. doi:10.1029/2007GL031831

\section{Submit your manuscript to a SpringerOpen ${ }^{\circ}$ journal and benefit from:}

- Convenient online submission

- Rigorous peer review

- Immediate publication on acceptance

- Open access: articles freely available online

- High visibility within the field

Retaining the copyright to your article 